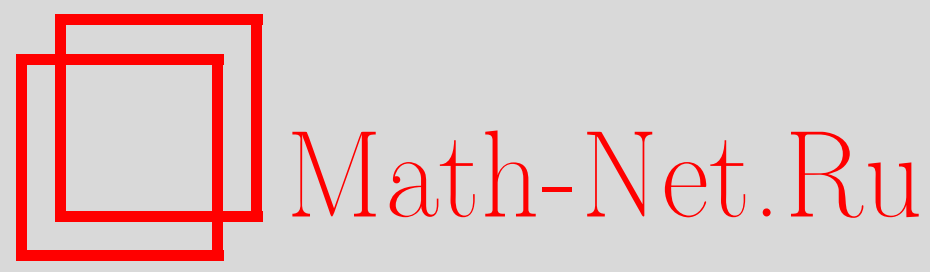

Л. Аккарди, М. Скайде, Реализации квадратичного белого шума и алгебры конечных разностей в гильбертовом модуле, Матем. заметки, 2000, том 68, выпуск 6, 803-818

DOI: https://doi.org/10.4213/mzm1003

Использование Общероссийского математического портала Math-Net.Ru подразумевает, что вы прочитали и согласны с пользовательским соглашением http://www.mathnet.ru/rus/agreement

Параметры загрузки:

IP: 54.237 .206 .68

26 апреля 2023 г., 14:42:18

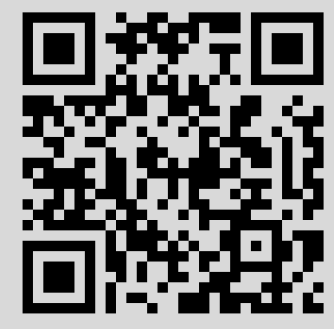




\title{
РЕАЛИЗАЦИИ КВАДРАТИЧНОГО БЕЛОГО ШУМА И АЛГЕБРЫ КОНЕЧНЫХ РАЗНОСТЕЙ В ГИЛЬБЕРТОВОМ МОДУЛЕ
}

\author{
Л. Аккарди, М. Скайде
}

\begin{abstract}
В статье развивается теория представлений для алгебры квадратов белого шума, основанная на конструкции гильбертова модуля. Мы приводим единственное фоковское представление и показываем, что пространством представления является обычное симметричное фоковское пространство. Хотя мы начинаем с рассмотрения систем с одной степенью свободы, мы заканчиваем статью теорией для систем с бесконечным иислом степеней свободы. Неожиданным обстоятельством является то, что построенное представление тесно связано с алгеброй конечных разностей Файнсильвера. Именно, существует голоморфное отображение алгебры конечных разностей в алгебру квадратов белого шума. Ограничение представления алгебры квадратов белого шума на этот образ совпадает с представлением Букаса на фоковском пространстве конечных разностей. Таким образом, мы расширяем представление Букаса на большую алгебру, порождаемую операторами рождения, уничтожения и числа частиц.

Библиографоия: 16 названий.
\end{abstract}

1. Введение. Следуя работе [1], под белым шумом мы понимаем операторнозначные распределения $b_{t}^{+}$и $b_{t}$ (параметризованные переменной $\left.t \in \mathbb{R}\right)$, которые удовлетворяют каноническим коммутационным соотношениям (KKC)

$$
\left[b_{t}, b_{s}^{+}\right]=\delta(t-s)
$$

Формально, квадраты белого шума должны быть операторнозначными распределениями $B_{t}^{+}=b_{t}^{+2}$ и $B_{t}=b_{t}^{2}$, удовлетворяюшими коммутационным соотношениям, вытекающим из КKC.

Однако, объекты $B_{t}^{+}, B_{s}$ оказьваются слишком сингулярными. Это проявляется в том, что их формальньй коммутатор имеет множитель $\delta^{2}(t-s)$, который априори не имеет смысла. Чтобы преодолеть эту трудность, в работе [1] было предложено рассматривать ренормализацию сингулярного объекта $\delta^{2}$, которая состоит в замене $\delta^{2}$ на $2 c \delta$, где $c>0$. Этот выбор мотивируется процедурой ренормализации, где $\delta$ аппроксимируется функциями $\delta_{\varepsilon}$ так, что $\delta_{\varepsilon}^{2} \rightarrow 2 c \delta$ в подходящем смысле, а постоянная $c$ может быть даже комплексной.

После ренормализации коммутатор $\left[B_{t}, B_{s}^{+}\right]=\delta(t-s)\left(2 c+4 b_{t}^{+} b_{t}\right)$ справа содержит операторную составляющую, именно оператор плотности числа частиц $N_{t}=b_{t}^{+} b_{t}$. Поскольку $\left[N_{t}, B_{s}^{+}\right]=\delta(t-s) 2 B_{t}^{+}$, мы получаем замкнутую алгебру Ли. Исключая

Работа второго автора выполнена при поддержке Deutsche Forschungsgemeinschaft. 
плотности с помошью соотношений $B_{f}^{+}=\int f(t) B_{t}^{+} d t$ и $N_{a}^{+}=\int a(t) N_{t}^{+} d t$ и вычисляя формально коммутаторы, мы получаем следующие соотношения:

$$
\begin{array}{lll}
{\left[B_{f}, B_{g}^{+}\right]=2 c \operatorname{Tr}(\bar{f} g)+4 N_{\bar{f} g},} & & f, g \in L^{2}(\mathbb{R}) \cap L^{\infty}(\mathbb{R}), \\
{\left[N_{a}, B_{f}^{+}\right]=2 B_{a f}^{+},} & a \in L^{\infty}(\mathbb{R}), \quad f \in L^{2}(\mathbb{R}) \cap L^{\infty}(\mathbb{R}),
\end{array}
$$

и $\left[B_{f}^{+}, B_{g}^{+}\right]=\left[N_{a}, N_{a^{\prime}}\right]=0$, где $\operatorname{Tr} f=\int f(t) d t$. Наша цель состоит в том, чтобы найти представление *-алгебры, порождаемой этими соотношениями.

В работе [1] представление этой алгебры было построено с помощью разложения Колмогорова для некоторого положительно определенного ядра. Наибольшая трудность состояла не в построении правильного ядра, а в доказательстве его положительности. В работе [2] Сниади нашел явную форму ядра, положительность которого очевидна. В настоящей работе мы идем другим путем, которьй мотивируется следующими соображениями. Соотношение (1.1a), выглядит как обычное КKC, за исключением того, что скалярное произведение в правой части принимает значения в алгебре, порождаемой операторами числа частиц $N_{a}$. Поэтому естественно попытаться реализовать его на гильбертовом модуле над алгеброй операторов числа частиц. Дополнительно на гильбертовом модуле можно реализовать также соотношение (1.1b), вводя явным образом левое умножение на операторы числа частиц.

Поскольку операторы числа частиц неограничены, мы не можем использовать теорию гильбертовых модулей на $C^{*}$-алгебрах, напротив, для этого потребуется теория предгильбетровых модулей над *-алгебрами в форме, предлагаемой в работе [3]. В п. 2 вводятся необходимые понятия. В п. 3 показано, как можно определить представления алгебры операторов числа частиц, фиксируя значения представления для $N_{a}$. Это важно для определения умножения слева и позволяет рассматривать алгебру, порождаемую $N_{a}$, не только как абстрактную алгебру, а как алгебру операторов числа частиц.

Основным является п. 4, где приводится конструкция двухстороннего предгильбертового модуля $E$ и показано, что над $E$ можно построить симметричньй фоковский модуль $\Gamma(E)$. Как мы увидим, естественные операторы рождения $a^{*}(f)$ на этом фоковском модуле и естественное умножение слева на $N_{a}$ удовлетворяют соотношению (1.1a) с точностью до аддитивных членов, а также соотношению (1.1b). С помощью конструкции тензорного произведения построено предгильбертово пространство, где вьполнены соотношения (1.1a), (1.1b). Это представление совпадает с конструкцией работы [1].

В п. 5 показано, что пространство представления изоморфно обычному симметричному фоковскому пространству над $L^{2}\left(\mathbb{R}, \ell^{2}\right)$. В последнем п. 6 показано, что это представление может рассматриваться как расширение представления Букаса для алгебры конечных разностей Файнсильвера [4] на фоковском пространстве конечных разностей. Исчисление алгебры квадратов белого шума обобщает исчисление Букаса [5]. В работе [6] Партасарати и Синха сконструировали алгебру конечных разностей с помощью операторов на симметричном фоковском пространстве. Однако, они не рассматривали вопрос об эквивалентности этого представления представлению Букаса. Вполне вероятно, что алгебра квадратов белого шума допускает аналогичное представление. Интересно также получить ответ на вопрос, является ли это представление точным.

2. Гильбертовы модули над *-алгебрами. Далеенам потребуется понятиегильбетрова модуля над *-алгеброй неограниченных операторов. Неограниченность затрудняет определение положительности. Для $C^{*}$-алгебры существует много эквивалентных 
способов определения положительных элементов и положительных линейных функционалов. В случае общих *-алгебр различные определения приводят к различньм понятиям положительности. Например, алгебраическое определение, при котором положительными элементами являются элементы, порождаемые вьпуклым конусом, которьй в свою очередь порождается всеми элементами вида $b^{*} b$, не содержит достаточный для наших целей запас положительных элементов. С другой стороны, слабое определение “элемент $b$ назьвается положительным, если $\varphi(b) \geqslant 0$ для всех положительньх функционалов $\varphi(\cdot)$ " является неконтролируемым, так как оно не позволяет показать, что скалярное произведение в тензорном произведении гильбертовых модулей также является положительным.

Здесь мы будем следовать работе [3], где некоторое образующее подмножество положительных элементов вводится аксиоматически и далее рассматривается порождаемьй ими конус. Такое определение положительности является контролируемьм, поскольку оно алгебраическое. Оно предполагает наличие левого умножения в двухстороннем гильбертовом модуле. Поэтому мы не вводим определения правого гильбертова модуля, а сразу определяем двухсторонний гильбертов модуль.

ОПРЕДЕЛЕниЕ 2.1. Пусть $\mathscr{B}$ - унитальная $*$-алгебра и пусть $S$ - некоторое выделенное образующее множество самосопряженных элементов из $\mathscr{B}$, содержащее 1 . Через $P(S)$ мы обозначаем выпукльй $\mathscr{B}$-конус, порождаемьй $S$ (т.е. множество всех сумм элементов из $a^{*} b a$, где $\left.b \in S, a \in \mathscr{B}\right)$. Будем говорить, что элементы из $P(S) S$-nоложительны.

Предгильбертовым $\mathscr{B}$-модулем назьвается $\mathscr{B}$ - $\mathscr{B}$-модуль $E$ (где $\mathbf{1} x=x \mathbf{1}=x$ ) с полуторалинейным скалярным произведением $\langle\cdot, \cdot\rangle: E \times E \rightarrow \mathscr{B}$, удовлетворяющим следуюшим условиям:

[i] $\langle x, x\rangle=0 \Longrightarrow x=0$ (определенность);

[ii] $\langle x, y b\rangle=\langle x, y\rangle b($ правосторонняя $\mathscr{B}$-линейность $)$;

[iii] $\langle x, b y\rangle=\left\langle b^{*} x, y\right\rangle(*$-свойство инволюиии $)$

и условию положительности, состоящему в том, что для любого $b \in S$ и любого конечного числа $x_{i} \in E$ существует конечное число $b_{k} \in S$ и $b_{k i} \in \mathscr{B}$ такие, что

$$
\left\langle x_{i}, b x_{j}\right\rangle=\sum_{k} b_{k i}^{*} b_{k} b_{k j} .
$$

Если условие определенности [i] нарушено, мы используем термины полускалярное произведение и полугильбертов модуль.

Отображение $a$ на полугильбертовом $\mathscr{B}$-модуле $E$ назьвается сопрягаемым, если существует отображение $a^{*}$ на $E$ такое, что $\langle x, a y\rangle=\left\langle a^{*} x, y\right\rangle$. Через $\mathscr{L}^{r}(E)$ и $\mathscr{L}^{a}(E)$ мы обозначаем пространства правых $\mathscr{B}$-линейных и сопрягаемых отображений на $E$ соответственно. Заметим, что на предгильбертовом модуле сопряжение единственно и что $\mathscr{L}^{a}(E) \subset \mathscr{L}^{r}(E)$.

Поскольку $1 \in S$, скалярное произведение $S$-положительно (т.е. $\langle x, x\rangle \in P(S)$ ), а поскольку $S$ состоит только из самосопряженных элементов, скалярное произведение симметрично (т.е. $\langle x, y\rangle=\langle y, x\rangle^{*}$ ) и антилинейно слева (т.е. $\langle x b, y\rangle=b^{*}\langle x, y\rangle$ ). Достаточно проверить положительность на подмножестве из $E$, которое порождает $E$ как правый модуль (см. [3]), либо доказать существование $b_{k} \in P(S)$. 
НАБлЮдЕниЕ 2.2. Любой полугильбертов модуль над коммутативной алгеброй $\mathscr{B}$ имеет тривиальную структуру левого модуля над $\mathscr{B}$, где правое и левое умножение одинаковы. Обозначим это тривиальное умножение через $b^{r}$, т.е. $b^{r}: x \mapsto x b$, для того чтобы различать этот случай от возможно нетривиального левого умножения.

Пусть $E$ и $F$ - полугильбертовы $\mathscr{B}$-модули. Их тензорное произведение над $\mathscr{B}$, выражаемое формулой $E \odot F=E \otimes F /\{x b \otimes y-x \otimes b y\}$, превращается в полугильбертов $\mathscr{B}$-модуль путем введения скалярного произведения $\left\langle x \odot y, x^{\prime} \odot y^{\prime}\right\rangle=\left\langle y,\left\langle x, x^{\prime}\right\rangle y^{\prime}\right\rangle$. В общем случае $E \odot F$ и $F \odot E$ могут быть весьма различньми объектами (например, см. [7, пример 6.7]). Обычно отображение $x \odot y \mapsto y \odot x$ не определяется корректно. Поэтому не всегда возможно сконструировать симметричный фоковский модуль. Знак тензора $\odot$ является “прозрачньм" для элемента алгебры, т.е. $x \odot b y=x b \odot y$. Любой оператор $a \in \mathscr{L}^{a}(E)$ порождает корректно определенный оператор $a \odot \mathrm{id} \in \mathscr{L}^{a}(E \odot F)$. Это вложение, однако, не обязано быть инъективньм. Для операторов $a$ на $F$ ситуация не столь удобна. В общем случае мы можем определить id $\odot a$, если $a-$ билинейньй оператор, но это вложение также не обязательно инъективно.

В дальнейшем мы будем изучать полугильбертовы модули. В силу ряда соображений желательно иметь строго положительное скалярное произведение. Например, в отличие от полускалярного, скалярное произведение гарантирует единственность сопряженного оператора. Мы вводим процедуру факторизации, позволяющую сконструировать предгильбертов модуль по заданному полугильбертовому модулю, если на $\mathscr{B}$ существует разделяющее множество $S^{*}$ положительных функционалов, совместное со структурой положительности, определяемой множеством $S$. Будем говорить, что функционал $\varphi$ на $\mathscr{B} S$-положителен, если $\varphi(b) \geqslant 0$ для всех $b \in P(S)$. Пусть $S^{*}$ - множество $S$-положительных функционалов. Будем говорить, что $S^{*}$ разделяет точки или является разделяющим, если условие $\varphi(b)=0$ влечет $b=0$ для всех $\varphi \in S^{*}$. Если $S^{*}$ является разделяющим множеством $S$-положительных функционалов на $\mathscr{B}$, то множество $\mathscr{N}=\{x \in E:\langle x, x\rangle=0\}$ является двухсторонним $\mathscr{B}$-подмодулем $E$. Кроме того, по определению фактор модуль $E_{0}=E / \mathscr{N}$ наследует структуру предгильбертова $\mathscr{B}$-модуля $\langle x+\mathscr{N}, y+\mathscr{N}\rangle=\langle x, y\rangle$.

Перед тем как перейти к фоковским модулям, мы опишем конструкцию, связьвающую наше алгебраическое определение положительности с "конкретной” положительностью операторов на предгильбертовых пространствах. Пусть $\pi$ - представление $\mathscr{B}$ на предгильбертовом пространстве $G$. Иньми словами, $G$ является $\mathscr{B}$ - $\mathbb{C}$-модулем. Снабжая $\mathbb{C}$ структурой вьпуклого $\mathbb{C}$-конуса, порождаемого положительным элементом $\mathbf{1}$, и продолжая естественным образом наше определение предгильбетрова модуля до двухстороннего модуля над разными алгебрами, можно поставить вопрос, является ли $G$ предгильбертовым модулем с естественным скалярньм произведением. Для этого необходимо и достаточно, чтобы $\langle g, \pi(b) g\rangle \geqslant 0$ для всех $g \in G$ и всех $b \in S$ (см. [3]). Если представление $\pi$ имеет это свойство, мы будем говорить, что $\pi$ является $S$-положительным. В частности, $\pi$ является $S$-положительньм, если $\pi$ отображает элемент из $S$ в сумму элементов вида $b^{*} b\left(b \in \mathscr{L}^{a}(G)\right)$.

Пусть теперь $\pi S$-положительно и пусть $E$ - предгильбертов $\mathscr{B}$-модуль. Заметим, что $\mathrm{id}_{\mathbb{C}}$ образует разделяющее множество положительных функционалов на $\mathbb{C}$. Конструкция тензорного произведения сохраняет силу, и мы получаем предгильбертов $\mathscr{B}$ - $\mathbb{C}$ модуль $H=E \odot G$. Иными словами, $H$ является предгильбертовым пространством с представлением $\rho(b)=b \odot \mathrm{id}$ для $\mathscr{B}$. Действительно, определение $\rho$ продолжается 
до представления $\mathscr{L}^{a}(E)$ на $H$. Дополнительно мы можем интерпретировать элементы $x \in E$ как отображения $L_{x}: g \mapsto x \odot g$ в $\mathscr{L}^{a}(G, H)$ с сопряженньми $L_{x}^{*}: y \odot g \mapsto \pi(\langle x, y\rangle) g$. Разумеется, $L_{b x b^{\prime}}=\rho(b) L_{x} \pi\left(b^{\prime}\right)$. Заметим, что отображение $x \mapsto L_{x}$ взаимно однозначно, если $\pi$ точное. В этом случае состояние $\rho$ является точным.

ОПРЕДЕЛЕНИЕ 2.3. Полным фоковским модулем над двухсторонним предгильбертовым $\mathscr{B}$-модулем $E$ называется двусторонний предгильбертов $\mathscr{B}$-модуль $\mathscr{F}(E)=$ $\bigoplus_{n=0}^{\infty} E^{\odot n}$ (где $E^{\odot 0}=\mathscr{B}$ со скалярньп произведением $\left\langle b, b^{\prime}\right\rangle=b^{*} b^{\prime}$ и алгебраической прямой суммой). Единицу $E^{\odot 0}$ мы обозначим через $\omega$, чтобы отличать ее от правого и левого умножения на $\mathbf{1} \in \mathscr{B}$.

На $\mathscr{F}(E)$ для каждого $x \in E$ определим оператор рождения $\ell^{*}(x)$ :

$$
\ell^{*}(x) x_{n} \odot \cdots \odot x_{1}=x \odot x_{n} \odot \cdots \odot x_{1}, \quad \ell^{*}(x) \omega=x,
$$

и сопряженный оператор уничтожсения

$$
\ell(x) x_{n} \odot \cdots \odot x_{1}=\left\langle x, x_{n}\right\rangle x_{n-1} \odot \cdots \odot x_{1}, \quad \ell(x) \omega=0 .
$$

Для каждого билинейного оператора $a$ на $E$ мы определим оператор $\lambda(a)$ на $\mathscr{F}(E)$, полагая

$$
\begin{gathered}
\lambda(a) x_{n} \odot \cdots \odot x_{1}=a x_{n} \odot x_{n-1} \odot \cdots \odot x_{1}+x_{n} \odot a x_{n-1} \odot \cdots \odot x_{1} \\
+\cdots+x_{n} \odot x_{n-1} \odot \cdots \odot a x_{1}
\end{gathered}
$$

и $\lambda(a) \omega=0$.

Фоковские модули впервые были рассмотрены в работах [8], [9]. Первое формальное определение полного фоковского модуля как гильбертова $C^{*}$-модуля было дано в работах [10], [11]. Следуя работе [3], мы используем здесь более общую конструкцию для *-алгебр.

3. Алгебра операторов числа частиц на $L^{\infty}(\mathbb{R})$. Рассмотрим коммутативную *-алгебру операторов числа частицна симметричном фоковском пространстве $\Gamma\left(L^{2}(\mathbb{R})\right)$, соответствующую элементам из $L^{\infty}(\mathbb{R})$. Как и в случае полного фоковского модуля, здесь используется алгебраическое определение симметричного фоковского пространства, а $\Gamma\left(L^{2}(\mathbb{R})\right)$ рассматривается как подпространство в $\mathscr{F}\left(L^{2}(\mathbb{R})\right)$.

ОПРЕДЕЛЕНИЕ 3.1. В полном фоковском пространстве $\mathscr{F}\left(L^{2}(\mathbb{R})\right)$ с вакуумным вектором $\Omega$ рассмотрим проекцию $P$

$$
P f_{n} \otimes \cdots \otimes f_{1} \stackrel{\text { def }}{=} \frac{1}{n !} \sum_{\sigma \in S_{n}} f_{\sigma(n)} \otimes \cdots \otimes f_{\sigma(1)}
$$

и $P \Omega=\Omega$. Симметричное фоковское пространство $\Gamma\left(L^{2}(\mathbb{R})\right)$ совпадает с $P \mathscr{F}\left(L^{2}(\mathbb{R})\right)$. Для $a \in L^{\infty}(\mathbb{R})$ определим оператор числа частиц $N_{a}=P \lambda(a) P$. Через

$$
\mathscr{N}=\operatorname{alg}\left\{N_{a}\left(a \in L^{\infty}(\mathbb{R})\right)\right\}
$$

мы обозначаем унитальную алгебру, порождаемую всеми $N_{a}$. 
Ясно, что $N_{a}^{*}=N_{a^{*}}$ и $N_{a} N_{a^{\prime}}=N_{a^{\prime}} N_{a}$; таким образом, $\mathscr{N}$ является коммутативной *-алгеброй. Из формулы разложения

$$
\frac{1}{2^{n}} \sum_{\varepsilon_{n}, \ldots, \varepsilon_{1}= \pm 1} \varepsilon_{n} \cdots \varepsilon_{1}\left(\varepsilon_{n} f_{n}+\cdots+\varepsilon_{1} f_{1}\right)^{\otimes n}=\sum_{\sigma \in S_{n}} f_{\sigma(n)} \otimes \cdots \otimes f_{\sigma(1)}
$$

следует, что вектор $\Omega$ и $f^{\otimes n}\left(f \in L^{2}(\mathbb{R}), n \in \mathbb{N}\right)$ образуют тотальное подмножество в $\Gamma\left(L^{2}(\mathbb{R})\right)$. Заметим также, что $N_{a}=P \lambda(a)=\lambda(a) P$. Это легко следует из равенства $P \lambda(a) P=\lambda(a) P$ и его сопряженного.

Пусть $I_{1}, I_{2}$ являются двумя непересекающимися измеримьпи подмножествами в $\mathbb{R}$. Обратим внимание на то, что хорошо известная формула факторизации

$$
\overline{\Gamma\left(L^{2}\left(S_{1}\right)\right) \otimes \Gamma\left(L^{2}\left(S_{2}\right)\right)} \cong \overline{\Gamma\left(L^{2}\left(S_{1} \cup S_{2}\right)\right)}
$$

сужается на алгебраическую область определения, так что

$$
\Gamma\left(L^{2}\left(S_{1}\right)\right) \otimes \Gamma\left(L^{2}\left(S_{2}\right)\right) \cong \Gamma\left(L^{2}\left(S_{1} \cup S_{2}\right)\right)
$$

(ср. доказательство теоремы 5.1). С учетом этого отождествления мы имеем $N_{\chi_{S_{1}}}=$ $N_{\chi_{S_{1}}} \otimes$ id и $N_{\chi_{S_{2}}}=$ id $\otimes N_{\chi_{S_{2}}}$. Аналогичные утверждения справедливы для факторизации на более чем два непересекающихся подмножества.

Поскольку $N_{a} \Omega=0$ для любого $a \in L^{\infty}(\mathbb{R})$, вакуумное состояние $\varphi_{\Omega}(\cdot)=\langle\Omega, \cdot \Omega\rangle$ является характером алгебры $\mathcal{N}$. Его ядро состоит из линейной оболочки всех мономов по крайней мере с одним сомножителем $N_{a}$, и его GNS-предгильбертово пространство есть $\mathbb{C} \Omega$.

В качестве подмножества $S$, задающего положительность на $\mathscr{B}$, мы выбираем

$$
S=\left\{N_{\chi_{I_{1}}} \ldots N_{\chi_{I_{n}}}: I_{i} \text { - ограниченные интервалы в } \mathbb{R}\left(n \in \mathbb{N}_{0}, i=1, \ldots, n\right)\right\} .
$$

ПРЕДЛОЖЕНИЕ 3.2. Определяющее представление id для $\mathscr{N}$ на $\Gamma\left(L^{2}(\mathbb{R})\right)$ является $S$-положительным.

ДокАЗАТЕЛЬСтво. Достаточно доказать, что $N_{\chi_{I}}$ имеет вид $\sum_{i} b_{i}^{*} b_{i}$, где $b_{i}$ выбираются (для всех $I)$ из коммутативной подалгебры в $\mathscr{L}^{a}\left(\mathscr{F}\left(L^{2}(\mathbb{R})\right)\right)$. Пусть $\lambda_{i}^{n}(a)$ $(n \in \mathbb{N}, 1 \leqslant i \leqslant n)$ - представление алгебры $L^{\infty}(\mathbb{R})$, которое действует на $i$-ю компоненту $n$-частичного подпространства в $\mathscr{F}\left(L^{2}(\mathbb{R})\right)$. Тогда

$$
N_{\chi_{I}}=\sum_{1 \leqslant i \leqslant n<\infty} \lambda_{i}^{n}\left(\chi_{I}\right)=\sum_{1 \leqslant i \leqslant n<\infty} \lambda_{i}^{n}\left(\chi_{I}\right)^{*} \lambda_{i}^{n}\left(\chi_{I}\right)
$$

В следующем пункте мы дадим определение представления $\mathscr{N}$, приписывая каждому $N_{a}$ оператор и расширяя его как гомоморфизм алгебр. Цель заключительной части нестоящего пункта состоит в том, чтобы показать, что это возможно по крайней мере, если мы ограничимся рассмотрением подалгебры $\mathfrak{S}(\mathbb{R})$ ступенчатых функций, которая плотна в $L^{\infty}(\mathbb{R})$ в подходящей слабой топологии.

Обозначим через $N$ оператор числа частиц на $\mathscr{F}\left(L^{2}(\mathbb{R})\right)$, который отображает $F \in$ $E^{\otimes n}$ в $n F$. В качестве $P N=N P$ мы используем тот же символ для оператора числа частиц на $\Gamma\left(L^{2}(\mathbb{R})\right)$. Тогда очевидно, что $\operatorname{alg}\{N\}$ изоморфна алгебре полиномов одной 
самосопряженной переменной. Более того, для каждого измеримого непустого множества $S \subset \mathbb{R}$ алгебра $\operatorname{alg}\left\{N_{\chi_{S}}\right\}$ изоморфна $\operatorname{alg}\{N\}$. Поэтому для любого самосопряженного элемента $a$ из $*$-алгебры $\mathscr{A}$ отображение $N \mapsto a$ продолжается до гомоморфизма $\operatorname{alg}\{N\} \rightarrow \mathscr{A}$.

Пусть $\mathfrak{t}=\left(t_{0}, \ldots, t_{m}\right)$ является точкой симплекса с $t_{0}<\cdots<t_{m}$. Тогда с помощью факторизации

$$
\Gamma\left(L^{2}\left(t_{0}, t_{m}\right)\right)=\Gamma\left(L^{2}\left(t_{0}, t_{1}\right)\right) \otimes \cdots \otimes \Gamma\left(L^{2}\left(t_{m-1}, t_{m}\right)\right)
$$

мы получаем

$$
\mathscr{N}_{\mathfrak{t}}:=\operatorname{alg}\left\{N_{\chi_{\left[t_{k-1}, t_{k}\right]}}(k=1, \ldots, m)\right\}=\operatorname{alg}\left\{N_{\chi_{\left[t_{0}, t_{1}\right]}}\right\} \otimes \cdots \otimes \operatorname{alg}\left\{N_{\chi_{\left[t_{m-1}, t m\right]}}\right\}
$$

Поэтому любое инволютивное отображение

$$
T_{\mathfrak{t}}: \mathfrak{S}_{\mathfrak{t}}(\mathbb{R}):=\operatorname{span}\left\{\chi_{\left[t_{k-1}, t_{k}\right]}(k=1, \ldots, m)\right\} \rightarrow \mathscr{A}
$$

с коммутативной областью значений однозначно определяет гомоморфизм $\rho_{\mathfrak{t}}: \mathscr{N}_{\mathfrak{t}} \rightarrow \mathscr{A}$, удовлетворяющий $\rho_{\mathfrak{t}}\left(N_{a}\right)=T_{\mathfrak{t}}(a)$.

Теперь можно доказать универсальное свойство алгебры $\mathscr{N}_{\mathfrak{S}}:=\bigcup_{\mathfrak{t}} \mathscr{N}_{\mathfrak{t}}$, которое состоит в том, что $\mathscr{N}_{\mathfrak{S}}$ является симметричной тензорной алгеброй над инволютивным векторньпм пространством $\mathfrak{S}(\mathbb{R})$.

Tеорема 3.3. Пусть $T: \mathfrak{S}(\mathbb{R}) \rightarrow \mathscr{A}$ - инволютивное отображсние $с$ коммутативной областью значений. Тогда существуют единственный гомоморфизм $\rho: \mathscr{N}_{\mathfrak{S}} \rightarrow \mathscr{A}$, удовлетворяющий условию $\rho\left(N_{a}\right)=T(a)$.

ДокАЗАТЕЛЬСТво. Достаточно заметить, что $\mathscr{N}_{\mathfrak{S}}$ является индуктивным пределом для $\mathscr{N}_{\mathfrak{t}}$ над множеством всех симплексов $\mathfrak{t}$, частично упорядоченных по включению. Обозначая через $\beta_{\mathfrak{t s}}$ каноническое вложение $\mathscr{N}_{\mathfrak{s}} \rightarrow \mathcal{N}_{\mathfrak{t}}(\mathfrak{s} \leqslant \mathfrak{t})$, нетрудно проверить, что $\rho_{\mathfrak{t}} \circ \beta_{\mathfrak{t s}}=\rho_{\mathfrak{s}}$. Другими словами, семейство $\rho_{\mathfrak{t}}$ однозначно продолжается как гомоморфизм $\rho$ на все $\mathcal{N}_{\mathfrak{S}}$.

4. Реализация алгебры квадратов белого шума. Идея реализации соотношений (1.1a) и (1.1b) на симметричном фоковском модуле состоит в том, чтобы принять правую часть (1.1a) как определение $\mathscr{N}$-значного скалярного произведения в модуле $E$, порождаемом элементами $f \in L^{2}(\mathbb{R}) \cap L^{\infty}(\mathbb{R})$, и затем определить левое умножение элементов $\mathscr{N}$ с порождающими элементами $f$, удовлетворяющими (1.1b). В то же время, попытка непосредственно использовать скалярное произведение (1.1a) оказывается неудачной. Поэтому мы используем линейный анзац (см. (4.1)) и затем далее подходящим образом подбираем константы.

С учетом теоремы 3.3 в настоящий момент мы ограничиваемся элементами из $\mathscr{N}_{\mathfrak{S}}$. Согласно (1.1а) это также ведет к необходимости ограничиться элементами $f \in \mathfrak{S}(\mathbb{R})$.

Для произвольных положительных постоянных $\beta$ и $\gamma$ на $\mathfrak{S}(\mathbb{R}) \otimes \mathcal{N}_{\mathfrak{S}}$ с естественной структурой правого $\mathscr{N}_{\mathfrak{S}}$-модуля мы определяем полуторалинейное отображение $\langle\cdot, \cdot\rangle$, полагая

$$
\langle f \otimes \mathbf{1}, g \otimes \mathbf{1}\rangle=M_{\bar{f} g}, \quad \text { где } \quad M_{a}=\beta \operatorname{Tr} a+\gamma N_{a},
$$

и продолжая его по (анти)линейности. 
Определим действие $M_{a}$ формулой

$$
M_{a}(f \otimes \mathbf{1}) \stackrel{\text { def }}{=} f \otimes M_{a}+\alpha a f \otimes \mathbf{1}
$$

с линейньм продолжением по правому сомножителю на все элементы из $\mathfrak{S}(\mathbb{R}) \otimes \mathscr{N}_{\mathfrak{S}}$. Здесь $\alpha$ - произвольная вешественная постоянная. Заметим, что скалярньй член $M_{a}$ не изменяет это коммутационное соотношение. Поэтому $N_{a}$ удовлетворяет коммутационным соотношениям с $\alpha$, замененными на $\alpha / \gamma$. Ввиду (1.1b) эта дробь должна быть равна 2 .

По определению умножение на $M_{a}$ слева является линейньм справа отображением на $\mathfrak{S}(\mathbb{R}) \otimes \mathscr{N}_{\mathfrak{S}} \cdot$ Нетрудно проверить, что $M_{a^{*}}=M_{a}^{*}$ является сопряженньп относительно полуторалинейного отображения (4.1). Согласно теореме 3.3 это определение действия слева распространяется на все элементы $\mathscr{N}_{\mathfrak{S}}$.

ПРЕДЛОЖЕНИЕ 4.1. Отображсене (4.1) является полускалярным произведением; поэтому $\mathfrak{S}(\mathbb{R}) \otimes \mathscr{N}_{\mathfrak{S}}$ является полугильбертовым $\mathscr{N}_{\mathfrak{S}}-$ модулем.

ДокАЗАТЕЛЬСТво.Достаточно проверить только условие положительности, потому что остальные свойства очевидны. Мы уже отмечали, что достаточно проверить положительность элементов из $\chi_{I_{i}} \otimes \mathbf{1}$, потому что эти элементы порождают $\mathfrak{S}(\mathbb{R}) \otimes \mathscr{N}_{\mathfrak{S}}$ как правьй модуль. Дополнительно мы можем предположить что $I_{i} \cap I_{j}=\varnothing$ для $i \neq j$. Тогда $\left\langle\chi_{I_{i}} \otimes \mathbf{1}, b\left(\chi_{I_{j}} \otimes \mathbf{1}\right)\right\rangle=0$ для $i \neq j$, каким бы ни был элемент $b \in \mathscr{N}_{\mathfrak{S}}$. Пусть теперь $b$ является элементом из $S$. После подходящей модификации отрезков $I_{i}$ мы можем предположить, что $b$ имеет вид $\prod_{i} N_{\chi_{i}}^{n_{i}}$, где $n_{i} \in \mathbb{N}_{0}$. Заметим, что

$$
N_{\chi_{I_{i}}}\left(\chi_{I_{j}} \otimes \mathbf{1}\right)=\left(\chi_{I_{j}} \otimes \mathbf{1}\right) N_{\chi_{I_{i}}}
$$

для $i \neq j$ и что

$$
N_{\chi_{I}}^{n}\left(\chi_{I} \otimes \mathbf{1}\right)=\sum_{k=0}^{n}\left(\begin{array}{l}
n \\
k
\end{array}\right)\left(\frac{\alpha}{\gamma}\right)^{(n-k)}\left(\chi_{I} \otimes N_{\chi_{I}}^{k}\right) .
$$

Доказательство проводится по индукции. Отсюда следует, что

$$
\left\langle\chi_{I_{i}} \otimes \mathbf{1}, b\left(\chi_{I_{j}} \otimes \mathbf{1}\right)\right\rangle=\delta_{i j} M_{\chi_{I_{i}}} \sum_{k=0}^{n_{i}}\left(\begin{array}{c}
n_{i} \\
k
\end{array}\right)\left(\frac{\alpha}{\gamma}\right)^{\left(n_{i}-k\right)} N_{\chi_{I_{i}}}^{k} \prod_{\ell \neq i} N_{\chi_{I_{\ell}}}^{n_{\ell}} .
$$

Определим $b_{k}=\left\langle\chi_{I_{k}} \otimes \mathbf{1}, b\left(\chi_{I_{k}} \otimes \mathbf{1}\right)\right\rangle$ и $b_{k i}=\delta_{k i} \mathbf{1}$. Тогда

$$
\left\langle\chi_{I_{i}} \otimes \mathbf{1}, b\left(\chi_{I_{j}} \otimes \mathbf{1}\right)\right\rangle=\sum_{k} b_{k i}^{*} b_{k} b_{k j},
$$

где $b_{k} \in P(S)$.

Выделим элементы нулевой длины такие, что

$$
E:=\mathfrak{S}(\mathbb{R}) \otimes \mathscr{N}_{\mathfrak{S}} / \mathscr{N}_{\mathfrak{S}(\mathbb{R}) \otimes \mathscr{N}_{\mathfrak{S}}}
$$

является двухсторонним предгильбертовьм $\mathscr{N}_{\mathfrak{S}}$-модулем. Обозначим

$$
f \otimes b+\mathscr{N}_{\mathfrak{S}(\mathbb{R}) \otimes \mathscr{N}_{\mathfrak{S}}}=f b
$$


Очевидно, что

$$
M_{a} f=f M_{a}+\alpha a f
$$

На подмножестве $f_{n} \odot \cdots \odot f_{1}$, порождающем $E^{\odot n}$, используя (4.2), мы получаем

$$
M_{a} f_{n} \odot \cdots \odot f_{1}=f_{n} \odot \cdots \odot f_{1} M_{a}+\alpha \lambda(a) f_{n} \odot \cdots \odot f_{1} .
$$

Поэтому на полном фоковском модуле $\mathscr{F}(E)$ вьполнено соотношение

$$
M_{a}=M_{a}^{r}+\alpha \lambda(a),
$$

где $M_{a}^{r}$ обозначает умножение на $M_{a}$ справа в смысле наблюдения 2.2 .

Теперь попытаемся определить симметричньй фоковский модуль над $E$ по аналогии с определением 3.1. Базис для симметризации порождается перестановками, изменяеюшими порядок сомножителей в тензоре $f \otimes g$. Как мы заметили ранее, мы не можем определить перестановки на $E \odot E$, отображая лишь $x \odot y$ в $y \odot x$ для всех $x, y \in E$. Мы можем, однако, сделать это так, как в работе [7] для иентрированных модулей, и определить перестановку только на таких $x, y$, которые получены для подходящего порождающего подмножества из $E$. Для построения общей перестановки на $E^{\odot n}$ мы также должны быть уверены, что перестановка является билинейной операцией.

ПРЕДЛОЖЕНИЕ 4.2. Отображсение

$$
\tau: f \odot g \mapsto g \odot f, \quad f, g \in \mathfrak{S}(\mathbb{R}) \subset E,
$$

единственным образом продолэсается по билинейности до унитарного изоморфизма $E \odot E \rightarrow E \odot E$ (т.е. оно сохраняет скалярное произведение и является сюрдективным отображением).

ДокАЗАТЕльство. Справедливо соотношение

$$
\begin{aligned}
\left\langle f \odot g, f^{\prime} \odot g^{\prime}\right\rangle & =\left\langle g,\left\langle f, f^{\prime}\right\rangle g^{\prime}\right\rangle=\left\langle g, M_{\bar{f} f^{\prime}} g^{\prime}\right\rangle=\left\langle g, g^{\prime} M_{\bar{f} f^{\prime}}+\alpha \bar{f} f^{\prime} g^{\prime}\right\rangle \\
& =M_{\bar{f} f^{\prime}} M_{\bar{g} g^{\prime}}+\alpha M_{\overline{g f} f^{\prime} g^{\prime}}=M_{\bar{g} g^{\prime}} M_{\bar{f} f^{\prime}}+\alpha M_{\overline{f g} g^{\prime} f^{\prime}}=\left\langle g \odot f, g^{\prime} \odot f^{\prime}\right\rangle .
\end{aligned}
$$

Элементы $f \odot g$ составляют (правое) образующее подмножество в $E \odot E$. Поэтому $\tau$ продолжается как изометрическое отображение на $E \odot E$. Очевидно, что это продолжение сюръективно, так что отображение $\tau$ является унитарньг.

Остается доказать, что отображение $\tau$ билинейно. Вновь достаточно проверить это свойство на образуюшем подмножестве и проверить его только для генераторов $M_{a}$. Имеем

$$
\begin{aligned}
\tau\left(M_{a} f \odot g\right) & =\tau\left(f \odot g M_{a}+\alpha(a f \odot g+f \odot a g)\right) \\
& =g \odot f M_{a}+\alpha(g \odot a f+a g \odot f)=M_{a} g \odot f=M_{a} \tau(f \odot g)
\end{aligned}
$$

Теперь можно определить симметричный фоковский модуль $\Gamma(E)$ так же, как и в определении 3.1. Предыдущее предложение показывает, что $P M_{a}=M_{a} P$, т.е. $P$ является билинейной проекцией. Как и ранее, мы имеем $P \lambda(a)=\lambda(a) P=P \lambda(a) P$. Следовательно, соотношение (4.3) также остается справедливьг на симметричном фоковском модуле. В дальнейшем мы не будем различать $\lambda(a)$ и его сужение на $\Gamma(E)$. В обоих 
случаях мы обозначаем оператор числа частиц через $N:=\lambda(\mathbf{1})$. Так как отображение $\lambda(a)$ билинейно, $N$ также обладает этим свойством и $N P=P N$. Из (3.1) следует, что симметричные тензоры составляют образующее подмножество.

Для любого $x \in E$ мы определяем оператор рождения на $\Gamma(E)$ как $a^{*}(x)=\sqrt{N} P \ell^{*}(x)$. Очевидно, что $x \mapsto a^{*}(x)$ является билинейным отображением, так как отображение $x \mapsto \ell^{*}(x)$ обладает этим свойством. Справедливы коммутационные соотношения

$$
M_{a} a^{*}(f)=a^{*}\left(M_{a} f\right)=a^{*}\left(f M_{a}+\alpha a f\right)=a^{*}(f) M_{a}+\alpha a^{*}(a f) .
$$

Разумеется, $a^{*}(x)$ имеет сопряженньй, именно, $a(x)=\ell(x) P \sqrt{N}$.

Рассмотрим теперь операторы рождения $a^{*}(f)$ и уничтожения $a(f)$ на элементе $f$ из $\mathfrak{S}(\mathbb{R}) \subset E$. Их действие на симметричных тензорах $g^{\odot n}(g \in \mathfrak{S}(\mathbb{R}))$ имеет вид

$$
a^{*}(f) g^{\odot n}=\frac{1}{\sqrt{n+1}} \sum_{i=0}^{n} g^{\odot i} \odot f \odot g^{\odot(n-i)}, \quad a(f) g^{\odot n}=\sqrt{n} M_{\bar{f} g} g^{\odot(n-1)}
$$

Очевидно, $a^{*}(f) a^{*}(g)=a^{*}(g) a^{*}(f)$. В тоже время, аналогичные соотношения неверны для $a^{*}(x)$ и $a^{*}(y)$ для произвольных элементов $x, y \in E$.

Для ККС нам нужно вычислить $a(f) a^{*}\left(f^{\prime}\right)$ и $a^{*}\left(f^{\prime}\right) a(f)$. Имеем

$$
\begin{aligned}
a(f) a^{*}\left(f^{\prime}\right) g^{\odot n} & =\frac{1}{\sqrt{n+1}} a(f) \sum_{i=0}^{n} g^{\odot i} \odot f \odot g^{\odot(n-i)} \\
& =M_{\bar{f} f^{\prime}} g^{\odot n}+M_{\bar{f} g} \sum_{i=0}^{n-1} g^{\odot i} \odot f^{\prime} \odot g^{\odot(n-1-i)}
\end{aligned}
$$

И

$$
\begin{aligned}
a^{*}\left(f^{\prime}\right) a(f) g^{\odot n} & =\sqrt{n} a^{*}\left(f^{\prime}\right) M_{\bar{f} g} g^{\odot(n-1)}=\sqrt{n}\left(M_{\bar{f} g} a^{*}\left(f^{\prime}\right)-\alpha a^{*}\left(\bar{f} g f^{\prime}\right)\right) g^{\odot(n-1)} \\
& =M_{\bar{f} g} \sum_{i=0}^{n-1} g^{\odot i} \odot f^{\prime} \odot g^{\odot(n-1-i)}-\alpha \lambda\left(\bar{f} f^{\prime}\right) g^{\odot n}
\end{aligned}
$$

После суммирования суммы по $i$ исчезают. Учитывая произвольность $g^{\odot n}$, а также соотношение (4.3), мы получаем

$$
\begin{aligned}
{\left[a(f), a^{*}\left(f^{\prime}\right)\right] } & =M_{\bar{f} f^{\prime}}+\alpha \lambda\left(\bar{f} f^{\prime}\right)=2 M_{\bar{f} f^{\prime}}-M \bar{r} f^{\prime} \\
& =\beta \operatorname{Tr}\left(\bar{f} f^{\prime}\right)+2 \gamma N_{\bar{f} f^{\prime}}-\gamma N_{\bar{f} f^{\prime}}
\end{aligned}
$$

Иными словами, полагая $\beta=2 c$ и $\gamma=2$, мы реализовали (1.1a) операторами $a^{*}(f)$, однако лишь по модулю умножения справа на некоторьй элемент из $\mathscr{N}_{\mathfrak{S}}$. Заметим, что эта конструкция не зависит от выбора $\alpha$. Полагая $\alpha=4$, мы реализуем также соотношение (1.1b).

Таким образом, мы должны сделать две веши. Во-первых, нужно избавиться от вклада от $N_{a}^{r}$ в предыдущих соотношениях. Во-вторых, чтобы сравнить с конструкцией алгебры из работы [1], мы должны дать интерпретацию нашей конструкции алгебры в 
терминах предгильбертовых пространств. Обе цели можно достичь одновременно следуюшим образом.

Рассмотрим тензорное произведение $H=\Gamma(E) \odot \mathbb{C} \Omega$ для $\Gamma(E)$ с предгильбертовьм $\mathscr{N}_{\mathfrak{S}}$-C-модулем $\mathbb{C} \Omega$, которьй является предгильбертовым пространством, снабженным ГНС-представлением, порождаемьм вакуумным состоянием $\varphi_{\Omega}$ на $\mathscr{N}_{\mathfrak{S}}$. Такое тензорное произведение допустимо, потому что любоеподпредставление на инвариантном подпространстве $S$-положительно согласно предложению 3.2 , определяющему представление $\mathcal{N}$ на $\Gamma\left(L^{2}(\mathbb{R})\right)$. Таким образом, $H$ является предгильбертовым пространством, которое имеет представление $\mathscr{L}^{a}(\Gamma(E))$. В этом представлении все операторы $N_{a}^{r}$ представляются нулем. Действительно, $N_{a}^{r}$ коммутируют со всеми операторами, поэтому мы ставим их справа и имеем

$$
N_{a}^{r} g^{\odot n} \odot \Omega=g^{\odot n} \odot N_{a}^{r} \Omega=0 .
$$

Обозначим через $B_{f}^{+}$образ $a^{*}(f)$ в $\mathscr{L}^{a}(H)$. Образ $N_{a}$ совпадает с образом $4 \lambda(a)$. Обозначим тем же символом оператор $N_{a}$. Через $\Phi=\omega \odot \Omega$ мы обозначаем вакуумный вектор в $H$.

Теорема 4.3. Oператоры $B_{f}^{+}, N_{a} \in \mathscr{L}^{a}(H)(f, a \in \mathfrak{S}(\mathbb{R}))$ удовлетворяют соотношениям (1.1a), (1.1b) $u\left[B_{f}^{+}, B_{g}^{+}\right]=\left[N_{a}, N_{a^{\prime}}\right]=0$. Кроме того, линейная оболочка векторов $B_{f}^{+n} \Phi\left(f \in \mathfrak{S}(\mathbb{R}), n \in \mathbb{N}_{0}\right)$ плотна в $H$.

ЗАмЕчАнИЕ 4.4. Ясно, что $H$ является именно предгильбертовым пространством в смысле конструкции работы [1]. Однако, в работе [1] скалярноепроизведениена тотальном множестве векторов было определено априори и было весьма сложно проверить их положительность. Здесь же положительность и корректная определенность представления обеспечиваются автоматически.

ЗАмЕчание 4.5. Полагая $H_{n}=\operatorname{span}\left\{B_{f}^{+n} \Phi(f \in \mathfrak{S}(\mathbb{R}))\right\}$, мы видим, что в обозначениях [3] $H=\bigoplus_{n=0}^{\infty} H_{n}$ является интерактивным фоковским пространством с операторами рождения $B_{f}^{+}$из работы [12].

ТЕОремА 4.6. Представления соотношений (1.1a) и (1.1b) операторами на $\bigoplus_{n=0}^{\infty} \overline{H_{n}}$ распространяются на әлементы $f \in L^{2}(\mathbb{R}) \cap L^{\infty}(\mathbb{R})$ и $а \in L^{\infty}(\mathbb{R})$.

ДокАЗАТЕльство. Мы распространим формально определение операторов $B_{f}^{+}$и $N_{a}$ на $f \in L^{2}(\mathbb{R}) \cap L^{\infty}(\mathbb{R})$ и $a \in L^{\infty}(\mathbb{R})$, рассматривая их как операторы на векторах вида $B_{f}^{+n} \Phi\left(f \in L^{2}(\mathbb{R}) \cap L^{\infty}(\mathbb{R}), n \in \mathbb{N}_{0}\right)$. Скалярное произведение векторов мы определяем с помощью продолжения скалярного произведения векторов, где $f \in \mathfrak{S}$ в $\sigma$-слабой топологии на $L^{\infty}(\mathbb{R})$. Такое продолжение, очевидно, корректно и единственно. Положительность этого скалярного произведения следует из свойств аппроксимируюших произведений и корректной определенности операторов, поскольку все используемые операторы имеют формальные сопряженные.

5. Ассоциированная мультипликативная система. Пусть $I \subset \mathbb{R}-$ конечное объединение интервалов. Обозначим через $H_{I}$ подпространство в пространстве $H$, образованное линейной оболочкой векторов вида $B_{f}^{+{ }^{n}} \Phi\left(f \in \mathfrak{S}(I), n \in \mathbb{N}_{0}\right)$. В частности, для $0 \leqslant t \leqslant \infty$ мы полагаем $H_{t}:=H_{[0, t)}$. Это означает, что $H_{0}=H_{\varnothing}=\mathbb{C} \Phi$. Заметим, что $H_{I}$ не зависит от того, являются ли интервалы из $I$ открытыми, полуоткрытыми или замкнутыми. 
Обозначим через $I+t$ сдвиг множества $I$ и через $f_{t}$ соответствуюший сдвиг функции: $f_{t}(s)=f(s-t)$. Очевидно, что, отображая $B_{f}^{+n} \Phi$ в $B_{f_{t}}^{+n} \Phi$, мы определяем изоморфизм $H_{I} \rightarrow H_{I+t}$.

Заметим, что согласно соотношению (1.1a) операторы $B_{f}$ и $B_{g}^{+}$, соответствующие функциям $f \in \mathfrak{S}(I)$ и $g \in \mathfrak{S}(\mathbb{R} \backslash I)$ коммутируют. Определим $\mathscr{N}_{I}:=\operatorname{alg}\left\{N_{a}(a \in \mathfrak{S}(I))\right\}$. Тогда в силу соотношения $(1.1 \mathrm{~b})$ элементы из $\mathscr{N}_{I}$ также коммутируют со всеми $B_{g}$, соответствующими функциям $g \in \mathfrak{S}(\mathbb{R} \backslash I)$.

ТЕОРема 5.1. Пусть $I, J \subset \mathbb{R}$ - конечные облединения интервалов, причем $I \cap J=\varnothing$. Тогда

$$
U_{I J}: B_{f}^{+n} B_{g}^{+m} \Phi \mapsto B_{f}^{+n} \Phi \otimes B_{g}^{+m} \Phi, \quad f \in H_{I}, \quad g \in H_{J},
$$

продолжсается как изоморфизм $H_{I \cup J} \rightarrow H_{I} \otimes H_{J}$. Композиция таких изоморфизмов ассочиативна в том смысле, что

$$
\left(U_{I J} \otimes \mathrm{id}\right) \circ U_{(I \cup J) K}=\left(\mathrm{id} \otimes U_{J K}\right) \circ U_{I(J \cup K)}
$$

ДокАЗАТЕЛЬСТво. Ясно, что векторы $B_{f}^{+n} B_{g}^{+m} \Phi$ образуют тотальное множество в $H_{I \cup J}$ и множество векторов $B_{f}^{+n} \Phi \otimes B_{g}^{+m} \Phi$ также тотально в $H_{I} \otimes H_{J}$. Поэтому достаточно проверить изометричность. Имеем

$$
\left\langle B_{f}^{+n} B_{g}^{+m} \Phi, B_{f^{\prime}}^{+n^{\prime}} B_{g^{\prime}}^{+m^{\prime}} \Phi\right\rangle=\left\langle B_{g}^{+m} \Phi, B_{f}^{n} B_{f^{\prime}}^{+n^{\prime}} B_{g^{\prime}}^{+m^{\prime}} \Phi\right\rangle
$$

Без потери общности можно предположить, что $n \geqslant n^{\prime}$. Тогда

$$
B_{f}^{n} B_{f^{\prime}}^{+n^{\prime}}=B_{f}^{n-n^{\prime}} B_{f}^{n^{\prime}} B_{f^{\prime}}^{+n^{\prime}}=B_{f}^{n-n^{\prime}} \sum_{k=0}^{n^{\prime}} b_{k} B_{f^{\prime}}^{+k} B_{f}^{k}
$$

где $b_{k} \in \mathcal{N}_{I}$. Поскольку $B_{f}$ коммутирует с $B_{g}^{+}$и $B_{f} \Phi=0$, ненулевой вклад дают только $B_{f}^{n-n^{\prime}} b_{0}$. С другой стороны, $b_{0}$ также коммутирует с $B_{g}^{+}$и $b_{0} \Phi=\Phi \varphi_{\Omega}\left(b_{0}\right)=\Phi\left\langle\Phi, b_{0} \Phi\right\rangle$. Поэтому $B_{f}^{n-n^{\prime}}$ коммутирует с $B_{g}^{+}$и, действуя непосредственно на $\Phi$, дает 0 во всех случаях, кроме $n=n^{\prime}$. Таким образом, ненулевой вклад возникает только для $n=n^{\prime}$ и $m=m^{\prime}$. Мы получаем

$$
\begin{aligned}
\left\langle B_{f}^{+n} B_{g}^{+m} \Phi, B_{f^{\prime}}^{+n} B_{g^{\prime}}^{+m} \Phi\right\rangle & =\left\langle B_{g}^{+m} \Phi, B_{g^{\prime}}^{+m} \Phi\right\rangle\left\langle\Phi, b_{0} \Phi\right\rangle \\
& =\left\langle B_{g}^{+m} \Phi, B_{g^{\prime}}^{+m} \Phi\right\rangle\left\langle B_{f}^{+n} \Phi, B_{f^{\prime}}^{+n} \Phi\right\rangle,
\end{aligned}
$$

что и требовалось. 
СлЕДСтВИЕ 5.2. Справедливо соотношение $H_{s} \otimes H_{t} \cong H_{[0, s)+t} \otimes H_{t} \cong H_{s+t}$. Изоморфизмы $U_{s t}: H_{s} \otimes H_{t} \rightarrow H_{s+t}$ обладают свойством ассочиативной композиции; иными словами, $H_{t}$ образуют тензорное произведение предгильбертовых пространств в смысле [13].

Определение, данное в работе [13], чисто алгебраическое. Представляет интерес вопрос о том, образуют ли $\overline{H_{t}}$ тензорное произведение пространств в более сильном смысле Арвесона, который ввел понятие product system в [14]. В частности, для этого все пространства $\overline{H_{t}}$ должны быть сепарабельными бесконечномерными пространствами и должны вьполняться некоторые условия измеримости. Мы действуем менее прямолинейно.

Если $\overline{H_{t}}$ является системой Арвесона, то возникает вопрос, какой тип она имеет. Системами типа I являются в точности те, которые получаются из симметричного фоковского пространства. Характеристическим свойством симметричных фоковских пространств среди других систем Арвесона является то, что они порождаются линейной оболочкой экспоненциальных векторов. Иными словами, мы должны найти все семейства $\psi_{t} \in \overline{H_{t}}$ (так называемые $\left.е д и н и и ы\right)$, тензорная композиция которых имеет свойства, сходные с тензорньп произведением экспоненциальных векторов на индикаторньх функциях, $\psi_{s} \otimes \psi_{t}=\psi_{s+t}$.

Естественными кандидатами на роль экспоненциальных векторов являются

$$
\psi_{\rho}(t)=\sum_{n=0}^{\infty} \frac{B_{\rho \chi_{t}}^{+}{ }^{n} \Phi}{n !}=\sum_{n=0}^{\infty} \frac{\rho^{n}}{n !} B_{\chi_{t}}^{+{ }^{n}} \Phi
$$

где $\rho \in \mathbb{C}$ и $\chi_{t}:=\chi_{[0, t]}$. Если заменить $B^{+}$операторами рождения в обьчном симметричном фоковском пространстве, мы получим обычный экспоненциальный вектор $\psi\left(\rho \chi_{t}\right)$. Если $\psi_{\rho_{0}}(t)$ существует, то он является аналитической векторозначной функцией для $\rho:|\rho|<\left|\rho_{0}\right|$. Нетрудно проверить, что если существуют $\psi_{\rho}(s)$ и $\psi_{\rho}(t)$, то также существует вектор $\psi_{\rho}(s+t)$, равный $\psi_{\rho}(s) \otimes \psi_{\rho}(t)$ (см. следствие 5.2). Более того, $\psi_{\rho}(t)$ является аналитической функцией $\rho$, которую можно дифференцировать. Заметим, что

$$
B_{\chi_{t}}^{+{ }^{n}} \Phi=\left.\frac{d^{n}}{d \rho^{n}}\right|_{\rho=0} \psi_{\rho}(t)
$$

является замкнутой линейной оболочкой $\psi_{\rho}(t)\left(|\rho|<\left|\rho_{0}\right|\right)$. Поэтому если для каждого $t>0$ существует $\rho_{0}>0$ такое, что существует $\psi_{\rho_{0}}(t)$, то векторы $\psi_{\rho}(t)$ и их сдвиги образуют тотальное подмножество в $\overline{H_{\infty}}$.

Лемма 5.3. Если $|\rho|<1 / 2$, то существует $\psi_{\rho}(t)$. Более того,

$$
\left\langle\psi_{\rho}(t), \psi_{\sigma}(t)\right\rangle=e^{-(c t / 2) \ln (1-4 \bar{\rho} \sigma)}
$$

әде функиия

$$
\varkappa:(\rho, \sigma) \mapsto-\frac{c}{2} \ln (1-4 \bar{\rho} \sigma)
$$

является полохительно определенным ядром на $U_{1 / 2}(0) \times U_{1 / 2}(0)$. 
ДокАЗАТЕЛЬСтво. Сначала мы покажем, что левая часть (5.1) сушествует в более простом случае $\sigma=\rho$, что доказывает существование $\psi_{\rho}(t)$.

Положим $f=\rho \chi_{t}$. Тогда $(\bar{f} f) f=|\rho|^{2} f$. Отсюда следует коммутационное соотношение $N_{\bar{f} f} B_{f}^{+}=B_{f}^{+} N_{\bar{f} f}+2|\rho|^{2} B_{f}^{+}$. Более того, $2 c \operatorname{Tr}(\bar{f} f)=2 c|\rho|^{2} t$. Имеем

$$
\begin{aligned}
B_{f} B_{f}^{+n}= & B_{f}^{+} B_{f} B_{f}^{+n-1}+\left(2 c|\rho|^{2} t+4 N_{\bar{f} f}\right) B_{f}^{+n-1} \\
= & B_{f}^{+} B_{f} B_{f}^{+n-1}+B_{f}^{+n-1}\left(2 c|\rho|^{2} t+8|\rho|^{2}(n-1)\right)+B_{f}^{+n-1} 4 N_{\bar{f} f} \\
= & B_{f}^{+n} B_{f}+n B_{f}^{+n-1} 4 N_{\bar{f} f} \\
& +B_{f}^{+n-1} 2|\rho|^{2}((c t+4(n-1))+(c t+4(n-2))+\cdots+(c t+0)) \\
= & B_{f}^{+n} B_{f}+n B_{f}^{+n-1} 4 N_{\bar{f} f}+B_{f}^{+n-1} 2 n|\rho|^{2}(c t+2(n-1)) .
\end{aligned}
$$

Если применить этот оператор к вакуумному вектору $\Phi$, то первые два слагаемых исчезнут. Мы получаем рекуррентную формулу

$$
\frac{\left\langle B_{f}^{+n} \Phi, B_{f}^{+n} \Phi\right\rangle}{(n !)^{2}}=4|\rho|^{2}\left(\frac{c t}{2 n}+\frac{n-1}{n}\right) \frac{\left\langle B_{f}^{+n-1} \Phi, B_{f}^{+n-1} \Phi\right\rangle}{((n-1) !)^{2}}
$$

Очевидно, что ряд

$$
\sum_{n=0}^{\infty} \frac{\left\langle B_{f}^{+n} \Phi, B_{f}^{+n} \Phi\right\rangle}{(n !)^{2}}
$$

сходится, если и только если $4|\rho|^{2}<1$ или $|\rho|<1 / 2$.

Для фиксированного $\rho \in U_{1 / 2}(0)$ функция $\left\langle\psi_{\rho}(t), \psi_{\rho}(t)\right\rangle$ является равномерным пределом целых функций переменной $t$ и поэтому сама является целой функцией $t$. В частности, поскольку $\psi_{\rho}(s+t)=\psi_{\rho}(s) \otimes \psi_{\rho}(t)$, то должно существовать число $\varkappa \in \mathbb{R}$ (более точно из $\mathbb{R}_{+}$, потому что $\left.\left\langle\psi_{\rho}(t), \psi_{\rho}(t)\right\rangle \geqslant 1\right)$ такое, что $\left\langle\psi_{\rho}(t), \psi_{\rho}(t)\right\rangle=e^{\varkappa t}$. Значение $\varkappa$ находим, дифференцируя в точке $t=0$. Единственньй вклад в произведение

$$
\left.\frac{d}{d t}\right|_{t=0} 4|\rho|^{2}\left(\frac{c t}{2 n}+\frac{n-1}{n}\right) \cdots 4|\rho|^{2}\left(\frac{c t}{2}+0\right)
$$

дает правило Лейбница, если мы продифференцируем последний сомножитель и положим $t=0$ в оставшихся членах. Получаем

$$
\left.\frac{d}{d t}\right|_{t=0}\left\langle\psi_{\rho}(t), \psi_{\rho}(t)\right\rangle=\sum_{n=1}^{\infty}\left(4|\rho|^{2}\right)^{n} \frac{1}{n} \frac{c}{2}=-\frac{c}{2} \ln \left(1-4|\rho|^{2}\right) .
$$

Остальные утверждения вытекают из аналогичных рассуждений при замене $|\rho|^{2}$ на $\bar{\rho} \sigma$. Ясно, что $\bar{\rho} \sigma$ является положительно определенным ядром. Тогда в силу леммы Шура функция $\varkappa(\rho, \sigma)$ как предел линейных комбинаций степеней $\bar{\rho} \sigma$ также является положительно определенной. 
ЗАмЕЧАниЕ 5.4. Функция $\varkappa$ является ковариационной функцией произведения системы пространств в смысле Арвесона [14], которая определена на множестве всех единиц и сужена на множество специальных единиц $\psi_{\rho}(t)$. На множестве всех единиц необходимо учитывать множители $e^{c t}$ единиц. Ковариационная функция на этом двухпараметрическом множестве является лишь условно положительным ядром.

Пусть

$$
v_{\rho}=\sqrt{\frac{c}{2}}\left(2 \rho, \frac{(2 \rho)^{2}}{\sqrt{2}}, \ldots, \frac{(2 \rho)^{n}}{\sqrt{n}}, \ldots\right) \in \ell^{2} .
$$

Тогда $\left\langle v_{\rho}, v_{\sigma}\right\rangle=-(c / 2) \ln (1-4 \bar{\rho} \sigma)$ и векторы $v_{\rho}$ образуют тотальное множество в $\ell^{2}$. Иными словами, разложение Колмогорова для ковариационной функции является парой $\left(\ell^{2}, \rho \mapsto v_{\rho}\right)$. Следующая теорема является простым следствием леммы 5.3.

ТЕОрема 5.5. Существует единственный инвариантный относительно сдвигов изоморфизм $\overline{H_{\infty}} \rightarrow \overline{\Gamma\left(L^{2}\left(\mathbb{R}_{+}, \ell^{2}\right)\right)}$ такой, что

$$
\psi_{\rho}(t) \mapsto \psi\left(v_{\rho} \chi_{t}\right)
$$

Следовательно, $\overline{H_{t}}$ является мультипликативной системой Арвесона типа I.

ЗАмЕчАнИЕ 5.6. Определяя $E_{I}$ как подмодуль в $E$, порождаемый $\mathfrak{S}(I)$, мы получаем $\Gamma\left(E_{I \cup J}\right)=\Gamma\left(E_{I}\right) \odot \Gamma\left(E_{J}\right)$ для непересекающихся $I$ и $J$ так же, как и в доказательстве теоремы 5.1. Введенньй вьше изоморфизм отображает $a^{*}(f)^{n} a^{*}(g)^{m} \omega$ в $a^{*}(f)^{n} \omega \odot a^{*}(g)^{m} \omega$ и рассуждения те же, за исключением того, что они проще: $b \omega=\omega b$. Действительно, полагая $E_{t}=E_{[0, t)}$, мы получаем тензорное произведение системы $\left(\Gamma\left(E_{t}\right)\right)$ предгильбертовых $\mathscr{N}_{\mathfrak{S}}-\mathscr{N}_{\mathfrak{S}}$-модулей в смысле работы [13].

6. Связь с алгеброй конечных разностей. После замен $c \rightarrow 2$ и $\rho \rightarrow \rho / 2$ правая часть уравнения (5.1), продолженного очевидньм образом с множества индикаторных функций на множество ступенчатых функций, является ядром Букаса [15], [16], определяющим пространство представления для алгебры конечны pa [4]. Поэтому пространство Букаса и наше пространство совпадают.

После того, как установлено совпадение пространств представления, возникает естественньй вопрос о том, содержит ли алгебра квадратов белого шума элементы, удовлетворяющие соотношениям алгебры конечных разностей. Действительно, полагая $c=2$ и определяя

$$
Q_{f}=\frac{1}{2}\left(B_{f}^{+}+N_{f}\right), \quad P_{f}=\frac{1}{2}\left(B_{\bar{f}}+N_{f}\right), \quad T_{f}=1 \operatorname{Tr} f+P_{f}+Q_{f}
$$

для $f \in L^{2}(\mathbb{R}) \cap L^{\infty}(\mathbb{R})$, мы получаем

$$
\left[P_{f}, Q_{g}\right]=\left[T_{f}, Q_{g}\right]=\left[P_{f}, T_{g}\right]=T_{f g} .
$$

В частном случае $f=\bar{f} \in \mathfrak{S}$ эти соотношения являются соотношениями алгебры конечных разностей. Действительно, операторы $Q_{f}, P_{f}, T_{f}$ совпадают с операторами, найденньми Букасом. Однако не ясно, следуют ли соотношения $T_{f}=1 \operatorname{Tr} f+P_{f}+Q_{f}$ из (6.2) либо они независимы. Во втором случае представление Букаса не может быть точным.

Во всех случаях операторов $Q_{f}, P_{f}, T_{f}$ не достаточно, чтобы восстановить $B_{f}, B_{f}^{+}$, $N_{f}$. Можно лиш восстановить операторы $B_{f}^{+}-B_{f}$ и $B_{f}^{+}+B_{f}+2 N_{f}$. Поскольку алгебра квадратов белого шума порождается операторами рождения, уничтожения и числа частиц, представление алгебры конечных разностей должно порождаться некоторыми линейными комбинациями этих операторов. 


\section{СПИСОК ЦИТИРОВАННОЙ ЛИТЕРАТУРЫ}

[1] Accardi L., Lu Y.G., Volovich I. V. White Noise Approach to Classical and Quantum Stochastic Calculi. Preprint, Rome, to appear in the lecture notes of the Volterra International School of the same title, held in Trento, 1999.

[2] Sniady P. Quadratic Bosonic and Free White Noise. Preprint, 1999.

[3] Accardi L., Skeide M. Interacting Fock Space Versus Full Fock Module. Preprint, Rome, 1998. Revised in January 2000.

[4] Feinsilver P. J. Discrete analogues of the Heisenberg-Weyl algebra // Mh. Math. 1987. V. 104. P. 89-108.

[5] Boukas A. Stochastic calculus on the finite-difference Fock space // Quantum Probability and Related Topics / ed. L. Accardi. V. VI: World Scientific, 1991.

[6] Parthasarathy K. R., Sinha K. B. Unification of quantum noise processes in Fock spaces // Quantum Probability and Related Topics / ed. L. Accardi. V. VI: World Scientific, 1991. P. 371-384.

[7] Skeide M. Hilbert modules in quantum electrodynamics and quantum probability // Comm. Math. Phys. 1998. V. 192. P. 569-604.

[8] Accardi L., Lu Y. G. From the weak coupling limit to a new type of quantum stochastic calculus // Quantum Probability and Related Topics / ed. L. Accardi. V. VII: World Scientific, 1992. P. 1-14.

[9] Accardi L., Lu Y. G. The Wigner semi-circle law in quantum electrodynamics // Comm. Math. Phys. 1996. V. 180. P. 605-632.

[10] Pimsner M. V. A class of $C^{*}$-algebras generalizing both Cuntz-Krieger algebras and crossed products by $\mathbb{Z}$ // Free Probability Theory / ed. D. V. Voiculescu. V. 12: Fields Institute Communications, 1997. P. 189-212.

[11] Speicher R. Combinatorial Theory of the Free Product with Amalgamation and Operator-valued Free Probability Theory. Mem. Amer. Math. Soc. V. 627. Providence, R.I.: Amer. Math. Soc., 1998.

[12] Accardi L., Lu Y. G., Volovich I. V. Interacting Fock Spaces and Hilbert Module Extensions of the Heisenberg Commutation Relations. Kyoto: IIAS Publications, 1997.

[13] Bhat B. V. R., Skeide M. Tensor Product Systems of Hilbert Modules and Dilations of Completely Positive Semigroups. Preprint, Cottbus, Revised version, Rome. Submitted to InfiniteDimensional Analysis, Quantum Probability and Related Topics, 1999.

[14] Arveson W. Continuous Analogues of Fock Space. Mem. Amer. Math. Soc. V. 409. Providence, R.I.: Amer. Math. Soc., 1989.

[15] Boukas A. Quantum Stochastic Analysis: a Non-Brownian Case. PhD thesis: Southern Illinois University, 1988.

[16] Boukas A. An example of quantum exponential process // Mh. Math. 1991. V. 112. P. 209-215.

Center V. Volterra, Università degli Studi di Roma "Tor Vergata" (Л. Аккарди)

Lehrstuhl für Wahrscheinlichkeitstheorie und Statistik,

Brandenburgische Technische Universität Cottbus (М. Скайде)

E-mail: accardi@volterra.mat.uniroma2.it, skeide@math.tu-cottbus.de 